Plass, A.M.C., Timmermans, D.R.M., Wal, G. van der. Does stimulating self-care increase self-care behaviqur for minor illnesses of Dutch and Turkish inhabitants of a deprived area in The Netherlands? Patient Education and Counseling: 2006, 63(1-2), 97-103

\begin{tabular}{|l|l|}
\hline Postprint Version & 1.0 \\
\hline Journal website & http://www.pec-journal.com/article/S0738-3991(05)00269-7/fulltext \\
\hline Pubmed link & http://www.ncbi.nlm.nih.gov/pubmed/16242295 \\
\hline DOI & $10.1016 /$ j.pec.2005.09.005 \\
\hline
\end{tabular}

This is a NIVEL certified Post Print, more info at http://www.nivel.eu

\title{
Does stimulating self-care increase self-care behaviour for minor illnesses of Dutch and Turkish inhabitants of a deprived area in The Netherlands?
}

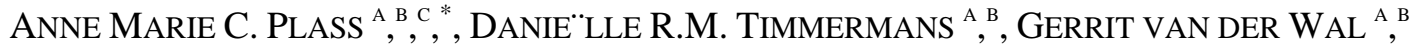 \\ ${ }^{a}$ Department of Public and Occupational Health, VU University Medical Center Amsterdam, The \\ Netherlands \\ ${ }^{\mathrm{b}}$ Institute for Research in Extramural Medicine, VU University Medical Center Amsterdam, The Netherlands \\ ${ }^{c}$ Department of Clinical Genetics and Human Genetics, Subdivision of Community Genetics, VU University \\ Medical Center, PO Box 7057, 1007 MB Amsterdam, Van der Boechorstraat 7, The Netherlands
}

\begin{abstract}
Objective: The aim of the present study was to examine whether self-care behaviour increases after a self-care stimulating intervention that proved to be successful in reducing care-seeking behaviour for minor illnesses of Turkish and Dutch inhabitants of a deprived area in the Netherlands, and to see whether there are cultural differences.

Method: This longitudinal study was based on a "pre-test/post-test one group" design. Data were collected during three structured face-to face interviews: before the intervention, and 6 months and 1 year after the intervention, in which GPs personally handed out booklets to their patients containing guidelines on the management of 12 minor illnesses.

Results: The number of self-reported self-care actions did not increase. In contrast to the Dutch, the Turkish participants reported a decrease in the number of self-care actions, their attitude towards self-care became more negative, and they perceived less control.

Conclusion: Apparently, a reduction in formal health care utilisation is not engendered by an increase in self-care behaviour. In order to make sure that interventions like these will have the intended effect, more research is needed, particularly among non-western populations.

Practice implications: In developing future healthcare-reducing interventions, one should be aware of possible unwanted side effects in non-western populations.
\end{abstract}

\section{INTRODUCTION}

Medical self-care is to be considered an integral part of the healthcare system for it is usually the first line of response to physical problems. Medical self--care, or selftreatment, can be defined as the range of behaviours undertaken by people to restore health when dealing with a medical problem, without professional intervention [1-3].

Furthermore, it is seen as a way to reduce formal healthcare utilisation [4].

A large number of interventions have been applied to stimulate medical self-care in an attempt to change care seeking behaviour [5-9]. Several studies have shown the effectiveness of medical self-care education in reducing health care utilisation [6-8], but others failed to do so [5,9].

In some studies, the success of a self-care stimulating intervention was measured by the decrease in the total number of consultations, while in other studies, success was measured by the decrease in the number of consultations for specific diseases, but self-care behaviour in itself was never a measure of success. Only 
Plass, A.M.C., Timmermans, D.R.M., Wal, G. van der. Does stimulating self-care increase self-care behaviqur for minor illnesses of Dutch and Turkish inhabitants of a deprived area in The Netherlands? Patient Education and Counseling: 2006, 63(1-2), 97-103

one study examined whether the intervention, aimed at stimulating self-care, actually led to an increase of self-care actions. Marklund et al. [4] found that participants in their intervention group (IG) had a greater use of over-the-counter drugs after the intervention, and read more medical books and pharmacy brochures in order to manage the medical problems themselves, compared to the control group (CG). Because utilisation of pharmacy brochures was part of the intervention, and because of the lack of a baseline measurement in this study, it is not evident whether self-care education actually led to an increase in selfcare behaviour. Summarizing, despite the large number of interventions that have been applied to stimulate medical selfcare in an attempt to change medical care-seeking behaviour, there is hardly any evidence to show that stimulating self-care leads to an increase in self-care behaviour, which in turn may lead to a reduction of formal healthcare utilisation.

The aim of the present study is to examine whether self care behaviour increases after an intervention aimed at reducing care-seeking behaviour by stimulating self-care behaviour. The focus is on minor illnesses (self-limiting health problems and minor ailments) because these comprise a considerable part of the workload of GPs, in particular in deprived areas with low economic and social standards and a high proportion of immigrants, e.g. Turkish people in the Netherlands [10,11]. Consultations for minor illnesses are often not very satisfactory for either the patient or the GP due to unmet expectations [12]. This might not only be due to language difficulties, but also to cultural differences [13]. In most cases, the GP can only offer reassurance whereas the patient desires treatment [14].

Decreasing the number of consultations for minor illnesses could therefore benefit both patients and GPs.

A booklet was compiled by local GPs in which the 12 most common minor illnesses of these specific patients were described in easy, understandable language. The booklet proved to be successful in decreasing the number of consultations for minor illnesses in Dutch and Turkish patients who were socially and economically deprived [15].

On the basis of self-report it was examined whether the intervention led to an increase in self-care activities in line with GP's advice, which was outlined in the booklet. The Theory of planned Behaviour (TpB) $[16,17]$ was applied to examine whether the factors that determine medical selfcare behaviour for minor illnesses changed as a result of the intervention. This theory is based on three aspects that explain health behaviour through behavioural intentions: attitude (e.g. health beliefs), social norms (e.g. social support) and perceived behavioural control (e.g. perceived barriers). All three aspects were found to be important factors in determining care-seeking behaviour [18,19], and is therefore used to examine self-care behaviour. Furthermore, since it is known that self-care behaviour differs between people, there might be culturally based differences in self-care behaviour as well. Therefore, the present study addresses the following questions: "Does a self-care stimulating intervention, aimed at reducing care-seeking behaviour, increase the number of self-care actions for minor illnesses, or change these so that they are more in line with GPs advice? Are there any differences between the Turkish and the Dutch low Socially and Economically Status (SES) participants with respect to self-care behaviour, its determinants, or behavioural change in self-care behaviour?’”

\section{METHOD}

\subsection{Design}

This longitudinal study was based on a "pre-test/post-test one group'” design. There was one pre-test (T0) before the intervention, and two post-tests: 6 months (T1) and 1 year after the intervention (T2). The pretest ( $T 0$ ) and second post-test (T2) were held during the winter, the first post-test (T1) was held in summer. Data were collected during structured face-to-face interviews with patients.

\subsection{Procedure}

Participants were recruited in the waiting rooms of seven different general practices, consisting of 13 GPs, that were located in a deprived area of The Hague, one of the biggest cities in the Netherlands. Dutch and Turkish interviewers recruited them, so each participant could be interviewed in his or her own language. Each patient who entered the waiting room, and who met the inclusion criteria, was invited by the interviewer to take part in the study. Inclusion was based on the GP's assessment of the frequency of consultation, which had to be 6 or more visits yearly, and a minimum age of 18 years. If the patient agreed, a short interview was held in the general practice, after which the patient participated in the intervention. Shortly after the first interview, a second complementary interview was held in the participant's house. This 
Plass, A.M.C., Timmermans, D.R.M., Wal, G. van der. Does stimulating self-care increase self-care behaviour for minor illnesses of Dutch and Turkish inhabitants of a deprived area in The Netherlands? Patient Education and Counseling: 2006, 63(1-2), 97-103

interview included additional questions (i.e. about demographic variables) that could not be asked in the general practice due to time limitations.

Thus, the first measurement (T0) consisted of two interviews.

Six months later (T1) and 1 year later (T2), respectively, a third and fourth interview took place in the participant's home.

\subsection{Intervention}

When the patients consulted their GP for a minor illness, the GP personally handed them a small booklet and gave them brief instructions on how to use it. The local GPs compiled this booklet in advance of the study, basing it on an existing, more extensive booklet [20]. The booklet contains guidelines on the management of 12 minor illnesses: 5 minor ailments (headache, stress, (low) back pain, insomnia and stomach-ache), and 7 self-limiting health problems (coughing, flu, diarrhoea, ear ache, children's diseases such as chicken pox, fever and sore throat). Each guideline consists of a brief description of the ailment and advice on when to seek professional help. Additionally, it contains suggestions for self-treatment. The booklets were also translated into the Turkish language.

\subsection{Measures}

Structured interviews were used to measure self-care behaviour, its determinants, and behavioural change in selfcare.

All questions were asked at baseline (T0), and most questions (except demographic variables) were repeated at time $1(T 1)$ and $2(T 2)$.

\subsubsection{Self-care}

In this study, self-report was used to measure self-care behaviour. McCauly et al. [21] reported in their study that the self-report questionnaire they used was shown to have a good internal reliability in detecting change from pre-test to posttest.

Klein et al. [18] found that adolescents'self-report of care is a valid method for determining health service utilization, and subjects were able to report accurately the care they had received after 5-7 months.

Therefore, we consider the selfreport measure we used to be reliable in detecting changes in self-reported self-care behaviour. Patients were asked whether they or their children had suffered from any of the 12 minor illnesses as described in the booklet during the 6 months preceding the interview. The minor illnesses were listed one by one by the interviewers and consisted of the 5 minor ailments, and 7 self-limiting health problems. If patients affirmed having suffered from the minor illness concerned, they were asked whether or not they had treated themselves, and if they had, what they had done in order to treat themselves. Patients could answer anything and as much as they liked, but the answers were scored by the interviewers in categories. These categories were based on self-care activities that were recommended by the 13 participating GPs, in addition to the category: “remaining”, which was categorised afterwards. The categories varied between minor illnesses. For example, categories for self-care in the case of headache were: getting enough rest, trying to relax, taking an aspirin, not taking food or drinks that might cause headache (like coffee or alcohol), talking to others about your worries, doing exercises, or "other"'. Categories for sore throat were: drinking, sucking on candy or something similar, gargling, taking a rest, taking an aspirin or ' 'other'. In addition, patients were asked whether or not they consulted their GP at a time that they or their children were suffering from minor illness.

\subsubsection{Determinants of self-care}

The questionnaire contained questions based on the Theory of planned Behaviour (TpB) [17] in order to measure determinants of behavioural change. Unless otherwise stated, all items were measured on a 5-point scale.

Attitude towards self-care was measured using six items, e.g. "There are many ailments for which you do not need to see a doctor, they cure themselves (totally disagree-totally agree)"' and 'Except in the case of a serious illness, it is better to take care of yourself than to seek professional help (totally disagree-totally agree)'”. The items were recoded. A score of 5 indicated a positive attitude towards self-care and a score of 1 indicated a negative attitude towards self-care.

Cronbach's alpha was 0.65 (relatively modest). No significant differences were found in the reliability of these sub-scales between Turkish and Dutch participants. 
Plass, A.M.C., Timmermans, D.R.M., Wal, G. van der. Does stimulating self-care increase self-care behaviour for minor illnesses of Dutch and Turkish inhabitants of a deprived area in The Netherlands? Patient Education and Counseling: 2006, 63(1-2), 97-103

The subjective norm was not measured since the booklet was targeted at attitude and perceived behavioural control, and not at the social norm. The booklet provided information on when to seek care, and gave suggestions for selftreatment.

As such, it did not directly mediate change in the subjective norm. Perceived behavioural control with regard to self-care was measured using three items, but one item was excluded because it did not match the other two based on scale analysis (it did not load on the same factor and reduced the reliability of the scale from 0.79 to almost zero: 0.05 ): "Do you find it hard to take care of yourself when you're suffering from a minor ailment? (always-never)", 'Do you have any doubts when treating yourself (alwaysnever)".

The items in this scale were recoded. A score of 5 indicated a high feeling of control over self-care and a score of 1 indicated a low feeling of control over self-care.

Cronbach's alpha was 0.79 . No significant differences in reliability were found between Turkish and Dutch participants.

Intention was measured only once, since this study was part of a longitudinal study in which self-reported behaviour was measured. Therefore, attitude and perceived behavioural control were directly related to the behaviour itself (see also Stroebe, 2000).

Further demographic variables were asked, e.g. nationality, marital status, age, education level, number of children, etc.

\subsection{Analyses}

MANOVAs were used to compare the mean scores from the three measurements within subjects. Therefore, only data on those participants who completed all interviews were included in the analyses. ANOVAs and paired T-tests were used to compare means between groups, i.e. Turkish versus Dutch participants.

The level of significance was 0.05 . All analyses were adjusted for practice $(N=7)$. No nesting effect of "practice" was found.

\subsection{Participants}

A total of 241 patients who were all inhabitants of a deprived area in The Hague were invited to participate in the study. 162 patients from seven different general practices (72 A.M.C. Plass et al. / Patient Education and Counseling 63 (2006) 97-103 99 Turkish, 70 Dutch and 20 of other nationalities) agreed and were included (79 refused). Allocation to the Dutch or the Turkish group was based on the patient's native country, together with their parent's native country, which was asked in the questionnaire. $27 \%$ of these patients suffered from a chronic disorder for which more than five visits yearly might be needed (lung diseases: $10.4 \%$; diabetes: $8.3 \%$; cardiovascular diseases: $8.3 \%$ ). There was no difference between participants and non-participants with regard to attendance frequency. People dropped out of the study after the first measurement ( $T 0$ ) for various reasons: e.g. change of GP, moving, remigration, and death. After 1 year, there were 117 patients remaining, which is a final response rate of $72 \%$ (based on the 162 patients that agreed to participate). Data from these individuals only are reported here. Participants had a mean age of 45 years (39 for the Turkish participants and 52 for the Dutch participants); the majority were women (80.1\%; 76\% among the Turkish participants; 83\% among the Dutch participants), were married (65.5\%; 85\% among the Turkish; 53\% among the Dutch), and had children (65.0\%; 79\% among the Turkish; 60\% among the Dutch).

Few Turkish participants were aged over $50(N=10)$.

Consistent with the characteristics of people living in socially and economically deprived areas, $69.2 \%$ had successfully completed, at most, lower vocational education (83\% among the Turkish; $61 \%$ among the Dutch) and 67\% were unemployed (74\% among the Turkish; 66\% among the Dutch). The patients that could no longer be contacted after the first measurement did not significantly differ with regard to demographic characteristics or outcome measures from the patients who completed all interviews.

\section{RESULTS}

Change in self-reported self-care behaviour is reported by accumulating all self-care actions that were reported for all 12 minor illnesses during each measurement, regardless of the illness. This measure is considered valid because no significant shift in illnesses reported before and after the intervention was found (see Table 1), except for insomnia and flu: Participants suffered significantly more from insomnia 
Plass, A.M.C., Timmermans, D.R.M., Wal, G. van der. Does stimulating self-care increase self-care behaviqur for minor illnesses of Dutch and Turkish inhabitants of a deprived area in The Netherlands? Patient Education and Counseling: 2006, 63(1-2), 97-103

after the intervention than before $(F(2,220) ; p=0.001)$, and significantly less from flu 1 year after the intervention than before or 6 months after the intervention $(F(2,220)=4,8 ; p=0.009)$.

Change in self-care actions for minor illnesses separately was not considered a valid measure, since the eligible numbers of patients who had suffered at least twice from the same minor illness during the research period were too small to be useful.

\subsection{Change in self-care}

The number of self-reported self-care actions did not change during the intervention, which in itself led to a significant decrease in self-reported care-seeking behaviour concerning minor illnesses in particular of the Turkish participants [15]. The number of self-care actions reported during the summer measurement (T1) was significantly less than the number reported during the pre-test $(T 0)$ and second post-test (T2), which were held during wintertime (see Table 2). Also, there were fewer minor illnesses reported during the summer measurement.

The majority of the self-reported self-care actions were in line with GP's advice (see Table 2).

\subsection{Differences between Turkish and Dutch}

\section{participants regarding self-care}

Only the number of self-care actions of the Dutch participants increased over time, particularly the number of self-care actions mentioned in the booklet, i.e. those in line with GP's advice. The number of self-care actions of the Turkish participants decreased over time, particularly the number of self-care actions in line with GP's advice. The number of self-care actions other than the ones mentioned in the booklet did not change during the research period (see Table 2).

\section{[TABLE 1]}

Overall, the Dutch participants used more self-care actions that were not advised in the booklet, compared to the Turkish participants, particularly when they suffered from headache $(F(1,53)=4.3 ; p=0.043)$, stress $(F(1,41)=8.0 ; p=0.007)$, and flu $(F(1,8)=4.6 ; p=0.013$., Turkish participants made significantly more use of self-care actions that were not advised in the booklet when they suffered from coughing $(F(1,26)=$ 5.8; $p=0.022$ ) compared to the Dutch.

Finally, Dutch participants used significantly more self-care actions to treat themselves for flu than the Turkish participants $(F(1,8)=11.2 ; p=0.010)$.

\subsection{Determinants of self-care behaviour}

No significant variation in attitude towards self-care behaviour was found after 1 year as compared to before the intervention. However, attitude towards self-care became significantly more positive towards self-care during the first 6 months of the study $(F(2,232)=11.6 ; p<0.001)$, but it became more negative during the second 6 months (see Table 3). Contrary to the Dutch, the attitude of the Turkish participants was already very positive towards self-care at the start of the study and did not change during the first 6 months. However, during the second 6 months it changed into an attitude that was significantly less positive towards self-care behaviour compared to their attitude at the start of the study.

\section{[TABLE 2 AND TABLE 3]}

A significant variation in perceived behavioural control over self-care was found $(F(2,232)=14.8 ; p<$ $0.001)$.

The perception of control over self-care particularly increased during the first 6 months of the study. The perception of control over self-care of the Turkish participants was significantly less compared to the Dutch participants during the entire study period $(F(1,101)=21.0 ; p<0.001)$, and it decreased significantly in the second 6 months in comparison to $T 1$, while the perception of control of the Dutch participants remained constant (see Table 3).

\section{DISCUSSION AND CONCLUSION}

\subsection{Discussion}

The data in this study clearly show that a reduction of formal health care utilisation for minor illnesses is not engendered by an increase in self-care behaviour. Although the goal of the intervention, which was 
Plass, A.M.C., Timmermans, D.R.M., Wal, G. van der. Does stimulating self-care increase self-care behaviour for minor illnesses of Dutch and Turkish inhabitants of a deprived area in The Netherlands? Patient Education and Counseling: 2006, 63(1-2), 97-103

decreasing the number of GP consultations for minor illnesses, was achieved [15], the number of selfreported self-care actions of the entire study population did not increase during the study period. People were already treating themselves when suffering from a minor illness, mainly in line with GP's advice.

There were striking differences between the Turkish and the Dutch participants in this study. The Turkish participants showed the strongest reduction of formal health care utilisation throughout the entire study period [15]. At the same time, they reported a decrease in the number of self-care actions. Furthermore, their attitude towards self-care became more negative and they also perceived less control over selfcare at the end of the study as compared to before the study.

These results are contrary to what was expected, for it is assumed that self-care behaviour needs to increase in order to decrease the number of GP consultations for minor illnesses.

The assumption that self-care behaviour needs to increase in order to decrease the number of GP consultations for minor illnesses does not seem to be valid. Contrary to the Turkish participants, the number of self-reported self-care actions of the Dutch increased, their attitude towards self-care became more positive, and their perception of control over self-care increased. However, the number of self-reported consultations for minor illnesses of the Dutch participants decreased only during the first 6 months of the study. An explanation for the differences found between the Turkish and the Dutch participants might be found in the kind of minor illnesses that participants reported having suffered from. A decrease in the number of self-care actions might also be due to a shift in the kind of minor illnesses. But analyses at the level of individual minor illnesses show no such shift. Another explanation for the decrease in self-care actions of the Turkish participants that was found might be that the booklet sometimes advises that it is best to do nothing, just towait for a couple of days or a fortnight until the symptoms disappear. Therefore, the decrease in self-care actions for minor illnesses could also be interpreted as a desired effect and success of the intervention. The Turkish participants might have taken the advice to do nothing more seriously than the Dutch, this is consistent with the findings of Uitewaal et al. and Kara and Asti who found that Turkish participants show strong adherence to patient education programmes [22,23]. Towait and see ("doing nothing'") was very often the first advice listed in the booklet, and could therefore possibly have been interpreted by the Turkish participants as being the preferred advice. This could have discouraged the Turkish participants to keep on treating themselves for self-care like they used to.

The advice of doing nothing might even have affected their attitude towards self-care, since self-care was not the first thing recommended. Furthermore, doing nothing might cause a feeling of less control over selfcare. If this is what really happened, one should be aware of the effect of the ranking order of advice, and it might be better to first give some advice to take some actions for self-care.

\subsection{Conclusion}

More research is needed to gain insight into possible unwanted side effects of interventions designed to stimulate self-care for the Turkish population. More information is needed on how non-western populations process information so that interventions like these have the intended effect.

\subsection{Practice implications}

In developing future interventions, one should be aware that the effectiveness of self-care stimulating interventions in order to reduce care-seeking behaviour would not necessarily increase self-care behaviour in itself. Apparently, there are other factors that bring about the effect.

Furthermore, it is important to be aware of possible, unwanted side effects when providing written information to non-western patients.

\section{REFERENCES}

[1] Dean K. Self-care components of lifestyles: the importance of gender, attitudes and the social situation. Soc Sci Med 1989;29: 137-52.

[2] Vickery DM. Medical self-care: a review of the concept and program models. Am J Health Promot 1986;1:23-8.

[3] Wykle ML, Haug MR. Multicultural ad social-class aspects of selfcare.

Generations 1993;17:25, 4.

[4] Marklund B, Almroth B, Schaffrath AM, Gunnarsson B, Ho" ijer B, Fridlund B. Promoting medical selfcare: evaluation of a family intervention implemented in the primary health care by pharmacies.

FamPract 1999;16:522-7. 
Plass, A.M.C., Timmermans, D.R.M., Wal, G. van der. Does stimulating self-care increase self-care behaviqur for minor illnesses of Dutch and Turkish inhabitants of a deprived area in The Netherlands? Patient Education and Counseling: 2006, 63(1-2), 97-103

[5] Heaney D, Wyke S, Wilson P, Elton R, Rutledge P. Assessment of impact of information booklets on use of healthcare services: randomised controlled trial. Br Med J 2001;322:1218-21.

[6] Chewning B, Sleath B. Medication decision-making and management: a client-centered model. Soc Sci Med 1996;42:389-98.

[7] Dean K. Self-care responses to illness: a selected review. Soc Sci Med [A] 1981;15:673-87.

[8] Kemper DW, Lorig K, Mettler M. The effectiveness of medical selfcare interventions: a focus on selfinitiated responses to symptoms.

Patient Educ Couns 1993;21:29-39.

[9] Little P, Somerville J, Williamson I, Warner G, Moore M, Wiles R, George S, Smith A, Peveler R.

Randomised controlled trial of self management leaflets and booklets for minor illness provided by post.

Br Med J 2001;322:1214-7.

[10] Grieve S. Measuring morale - does practice area deprivation affect doctors' well-being? $\mathrm{Br} \mathrm{J}$ Gen Pract 1997;47:547-52.

[11] Hassell K, Whittington Z, Cantrill J, Bates F, Rogers A, Noyce P.

Managing demand: transfer of management of self limiting conditions from general practice to community pharmacies. Br Med J 2001;323:146-7.

[12] Vingard E, Alfredsson L, Hagberg M, Kilbom A, Theorell T, Waldenstrom M, Hjelm EW, Wiktorin C, Hogstedt C. To what extent do current and past physical and psychosocial occupational factors explain care-seeking for low back pain in a working population? Results from the Musculoskeletal Intervention Center-Norrtalje Study.

Spine 2000;25:493-500.

[13] van Wieringen JC, Harmsen JA, Bruijnzeels MA. Intercultural communication in general practice. Eur J Public Health 2002;12: 63-8.

[14] Logghe K, Van Erp R, Kraetzer S. [Dialogue between Gp's and immigrants: good communication regains trust.] Versie: Tijdschrift voor gezondheid. Burgerschap en Politiek 1998;16:32-9.

[15] Plass AM, Timmermans DR, van derWal G. Decreasing the number of consultations for minor illnesses of Turkish and Dutch inhabitants of a deprived area in The Netherlands: an intervention study. FamPract 2005;22:51-7.

[16] Ajzen I. Attitudes, Personality, and Behavior. Milton Keynes: Open University Press; 1988.

[17] Ajzen I. The theory of planned behavior. Organizational behavior and human decision process 1991;50:179-211.

[18] Klein JD, Graff CA, Santelli JS, Hedberg VA, Allan MJ, Elster AB.

Developing quality measures for adolescent care: validity of adolescents' self-reported receipt of preventive services. Health Serv Res 1999;34:391-404.

[19] Moses S, Ngugi EN, Bradley JE, Njeru EK, Eldridge G, Muia E, Olenja J, Plummer FA. Health careseeking behavior related to the transmission of sexually transmitted diseases in Kenya. Am J Public Health 1994;84:1947-51.

[20] Van Dijk P. [In Dutch: The Home-Physician]. Utrecht: Kosmos-Z\&K Uitgevers B.V., 1997.

[21] Macaulay AP, Griffin KW, Botvin GJ. Initial internal reliability and descriptive statistics for a brief assessment tool for the life skills training drug-abuse prevention program. Psychol Rep 2002;91:459-62.

[22] Uitewaal PJ, Bruijnzeels MA, Bernsen RM, Voorham AJ, Hoes AW, Thomas S. Diabetes care in Dutch general practice: differences between Turkish immigrants and Dutch patients. Eur J Public Health 2004;14:15-8.

[23] Kara M, Asti T. Effect of education on self-efficacy of Turkish patients with chronic obstructive pulmonary disease. Patient Educ Couns 2004;55:114-20. 
Plass, A.M.C., Timmermans, D.R.M., Wal, G. van der. Does stimulating self-care increase self-care behaviqur for minor illnesses of Dutch and Turkish inhabitants of a deprived area in The Netherlands? Patient Education and Counseling: 2006, 63(1-2), 97-103

\section{TABLES}

Table 1

Percentages of respondents that reported having suffered from the minor illnesses mentioned during the 6 months preceding each interview

\begin{tabular}{lllr}
\hline & $T 0$ & $T 1$ & $T 2$ \\
\hline Headache & 73.5 & 76.9 & 74.4 \\
Stress & 67.5 & 65.0 & 63.2 \\
Coughing & 59.0 & 51.3 & 58.1 \\
(Low) back pain & 53.8 & 58.1 & 54.7 \\
Insomnia & 53.0 & 32.5 & 42.7 \\
Sore throat & 47.0 & 35.9 & 42.7 \\
Stomach ache & 46.2 & 46.2 & 36.8 \\
Flu & 43.6 & 33.3 & 55.6 \\
Diarrhoea & 20.5 & 19.7 & 20.5 \\
Fever & 13.7 & 10.3 & 9.4 \\
Earache & 6.8 & 13.7 & 13.7 \\
Children's disease & 1.7 & 3.4 & 2.6 \\
\hline
\end{tabular}

Note: $N=117, T 0=$ pre-test; $T 1=$ first post-test, 6 months after the intervention; $T 2=$ second post-test, 1 year after the intervention. 
Table 2

Mean number of self-reported minor illnesses and self-care actions; overall, in line with GP's advice, and other self-care actions, during the 6 months preceding each interview of the whole study population, the Dutch and the Turkish participants separately

\begin{tabular}{|c|c|c|c|c|}
\hline & Measurement & All $(N=117)$ & Dutch $(N=50)$ & Turkish $(N=54)$ \\
\hline \multirow[t]{3}{*}{ Mean number of different minor illnesses } & $T 0$ & 4.9 & 4.7 & 4.8 \\
\hline & $T 1$ & 4.5 & 4.4 & 4.3 \\
\hline & $T 2$ & 4.7 & 4.9 & 4.6 \\
\hline \multirow[t]{3}{*}{ Mean number of self-care actions } & $T 0$ & $5.3^{\mathrm{a}}$ & 5.1 & 5.3 \\
\hline & $T 1$ & $4.8^{\mathrm{b}}$ & 4.8 & 4.4 \\
\hline & $T 2$ & 5.2 & 5.9 & 4.2 \\
\hline \multirow[t]{3}{*}{ Mean number of self-care actions according to the booklet } & $T 0$ & 3.5 & 2.5 & $4.2^{\mathrm{c}}$ \\
\hline & $T 1$ & 3.0 & 2.2 & $3.7^{\mathrm{c}}$ \\
\hline & $T 2$ & 3.4 & 3.3 & 3.4 \\
\hline \multirow[t]{3}{*}{ Mean number of self-care actions other than mentioned in the booklet } & $T 0$ & 1.9 & 2.5 & $1.1^{\mathrm{c}}$ \\
\hline & $T 1$ & 1.8 & 2.6 & $0.8^{\mathrm{c}}$ \\
\hline & $T 2$ & 1.8 & 2.6 & $0.8^{\mathrm{c}}$ \\
\hline
\end{tabular}

Note: $T 0=$ baseline (before the intervention), $T 1=6$ months after the intervention; $T 2=1$ year after the intervention.

a Significantly different from $T 1$

b Significantly different from $T 2$.

c Turkish differ significantly from the Dutch at the same measurement.

Table 3

Mean scores on determinants of self-care behaviour

\begin{tabular}{|c|c|c|c|c|c|c|c|c|c|}
\hline & \multicolumn{3}{|l|}{ All } & \multicolumn{3}{|c|}{ Dutch } & \multicolumn{3}{|c|}{ Turkish } \\
\hline & To & $T 1$ & $T 2$ & $T 0$ & $T 1$ & $T 2$ & $T 0$ & $T 1$ & $T 2$ \\
\hline Attitude towards self-care & 3.4 & $3.7^{\mathrm{a}}$ & $3.3^{\mathrm{b}}$ & 3.3 & $3.6^{\mathrm{a}}$ & $3.3^{\mathrm{b}}$ & 3.6 & 3.7 & $3.4^{\mathrm{b}}$ \\
\hline Perceived behavioural control over self-care & 3.1 & $3.6^{\mathrm{a}}$ & $3.4^{\mathrm{c}}$ & 3.4 & $3.9^{\mathrm{a}}$ & $3.9^{\mathrm{c}}$ & 2.5 & $3.3^{\mathrm{a}}$ & $2.9^{\mathrm{b}, \mathrm{c}}$ \\
\hline
\end{tabular}

$T 0=$ pre-test; $T 1=$ first post-test, 6 months after the intervention; $T 2=$ second post-test, 1 year after the intervention.

a Significant difference between $T 0$ and $T 1$.

Significant difference between $T 1$ and $T 2$.

${ }^{c}$ Significant difference between $T 0$ and $T 2$. 